\title{
Discrete instability in nonlinear lattices
}

\author{
J. Leon, M. Manna \\ Physique Mathématique et Théorique, CNRS-UMR5825, \\ Université Montpellier 2, 34095 MONTPELLIER (France)
}

\begin{abstract}
The discrete multiscale analysis for boundary value problems in nonlinear discrete systems leads to a first order, strictly discrete, modulational instability (disappearing in the continuous envelope limit) above a threshold amplitude for wave numbers beyond the zero of group velocity dispersion. Applied to the electrical lattice [Phys. Rev. E, 51, 6127 (1995)], this acurately explains the experimental instability at wave numbers beyond 1.25 rad.cell $^{-1}$. The theory is also briefly discussed for the sine-Gordon and Toda lattices.
\end{abstract}

Introduction. The Benjamin-Feir instability describes the mechanism of the exponential growth of the modulation of a wave evolving in a continuous nonlinear dispersive medium [1,2]. It has revealed as a fundamental process occuring in various physical situations, from water waves to nonlinear optics through plasma waves, electrical transmission lines, etc..., see e.g. [3]. This instability, originally established for periodic wave trains (Stokes waves in surface water theory) [4, is actually a generic process in physics, well understood in the context of the nonlinear Schrödinger equation (NLS) 5] [6]. In the case on an infinite medium, and for the focusing NLS, this instability has been shown to evolve eventually into localized coherent nonlinear structures (the solitons), see e.g. [7].

The analysis of a nonlinear system is performed by first deriving from the original model a simpler limit equation which usually results to be the NLS. This universal limit is obtained by a very general approach called multiscale perturbation analysis [8]. It is worth remarking already that in such cases the instability is actually a second order effect: NLS is obtained as a first order perturbation and the Benjamin-Feir instability results from a perturbative analysis of NLS itself.

In the case of discrete systems where intrinsic nonlinear modes (called also discrete breathers) are known to exist [9]- [14], the modulational instability, mechanism for their spontaneous generation out of an initial disturbance, is usualy described in a semi-discrete multiscale analysis where the envelope of the discrete carrier wave is treated as a continuous function [15]- [17]. Fully discrete treatments have also been proposed as in 18.19, based on the rotating wave approximation which essentially consists in neglecting harmonics. In a nonlinear system, this procedure evidently may give wrong predictions for large times.

In some of these studies however, the problem is not that of the long time evolution of an initial perturbation but rather the far behavior of an input boundary disturbance (or forcing). This is particularly evident in the electrical transmission line of 17, 18 where an input signal (modulated wave) is sent at one end of the lattice and collected at various locations along the line.

Hence we consider here the problem of the scattering of a plane wave incoming into a nonlinear discrete medium.
Based on the method founded in [20], a discrete change of variables is introduced and used to construct the related discrete limit model. This results as a NLS-like equation in discrete space and with space and time exchanged. For wave numbers beyond the zero of the group velocity dispersion, it is shown that the waves are uniformly unstable as soon as their amplitudes exceed a threshold value. Moreover this instability is a purely discrete property as it is shown to disappear if the envelope is treated as a continuous function like in the semi-discrete approach.

This approach is applied to the electrical lattice of [18] where the problem is indeed that of the far behavior of an input boundary datum and for which a set of very detailed and acurate experimental results are displayed. In particular the measured threshold of modulational instability occurs at wave numbers beyond 1.25 (in rad.cell ${ }^{-1}$ ) which is the value predicted by the present theory. We predict also a threshold amplitude depending on the input carrier frequency and modulation. For one of the experiments of [18 this threshold value is $0.23 \mathrm{~V}$, significantly smaller than the amplitudes actually used in the experiments where damping had to be overcome. This threshold amplitude is shown to depend on the relative frequencies of the carrier v.s. modulation and hence can be easily varied which should motivate new experiments.

Basic tools. Treating a boundary value problem corresponds to making an expansion of the wave number around small deviations of the frequency from the linear dispersion law. To see this, we consider a plane wave

$$
A(n, t)=\exp [i(K n-\Omega t)]
$$

in a nonlinear lattice (with intersite spacing normalized to 1) with the linear dispersion relation of bandpass filter type

$$
\Omega^{2}=\omega_{0}^{2}+4 u_{0}^{2} \sin ^{2}(K / 2) .
$$

Due to nonlinearity, the actual frequency $\omega$ and wave number $k$ experience deviations form the values $\Omega$ and $K$ obeying the linear dispersion relation (22). There are two ways of representing these deviations.

With the wave packet

$$
u_{n}(t)=\int d k \hat{u}(k) e^{i(k n-\omega t)},
$$


we may expand the frequency in terms of the assumed small deviations from the value $K$ of the wave number as

$$
k=K+\epsilon \lambda, \quad \omega=\Omega+\epsilon v_{g} \lambda+\epsilon^{2} P \lambda^{2}+\cdots
$$

where $v_{g}=\partial \Omega / \partial K$ is the group velocity and $2 P=$ $\partial^{2} \Omega / \partial K^{2}$ the group velocity dispersion. In that case, the expression for the wave packet becomes

$$
u_{n}(t)=\epsilon e^{i(K n-\Omega t)} \phi(Z, T)
$$

where the slowly varying envelope $\phi$, namely

$$
\phi(Z, T)=\int d \lambda \hat{u}(\lambda) e^{i\left(\lambda Z-\lambda^{2} P T\right)}
$$

is written in the frame

$$
Z=\epsilon\left(n-v_{g} t\right), \quad T=\epsilon^{2} t
$$

This means that the effects of an initial disturbance are observed for large $\left(\epsilon^{-2}\right)$ times by following the waves at the group velocity and analyzing the perturbation about the plane wave solution. This approach then corresponds to treating initial value problems. Note in particular that the variable $Z$ hereabove defined becomes by construction a continuous variable.

Alternatively, considering the wave packet

$$
u_{n}(t)=\int d \omega \hat{u}(\omega) e^{i(k n-\omega t)},
$$

we may expand here the wave number in terms of the frequency as

$$
\omega=\Omega+\epsilon \nu, \quad k=K+\epsilon \frac{\nu}{v_{g}}+\epsilon^{2} Q \nu^{2}+\cdots
$$

(with $2 Q=\partial^{2} K / \partial \Omega^{2}$ ), hence giving

$$
u_{n}(t)=\epsilon e^{i(K n-\Omega t)} \psi(\xi, \tau)
$$

with the slow modulation

$$
\psi(\xi, \tau)=\int d \nu \hat{u}(\nu) e^{i\left(\nu^{2} Q \xi-\nu \tau\right)},
$$

in the frame

$$
\tau=\epsilon\left(t-n / v_{g}\right), \quad \xi=\epsilon^{2} n
$$

Such a reference frame has a clear physical meaning: one considers long distance $\left(\epsilon^{-2}\right)$ effects in the retarded time to let the input disturbance enough time to reach the observed lattice point. Hence this corresponds to the situation where the lattice is excited at one end, in other words to a boundary value problem. This is the tool we shall use hereafter when the variable $\xi$ is kept discrete as follows.
Discrete multiscaling. Performing a discrete multiscale analysis goes first with assuming a hypothesis of slow modulation. This means for the envelope $\psi_{n}(t)$ of the wave $A(n, t)$, that

$$
\left\|\partial_{t} \psi_{n}(t)\right\| \sim \epsilon\left\|\partial_{t} A(n, t)\right\|
$$

which then allows to chracterize the dimension $\epsilon$ of the slow variable $\tau$ by (12).

Second, as introduced in 20], we define a large grid indexed with the slow variable $m$ defined by sampling the original grid at each $N=\epsilon^{-2}$ (more precisely the closest odd integer). As a consequence we can index the variable $\xi_{n}$ by $m$ in the new grid, namely we can call $m$ a given point $n$ and $m+j$ the points $n+j N$ for all $j$. In short

$$
\cdots, \xi_{n-N}=m-1, \xi_{n}=m, \xi_{n+N}=m+1, \cdots .
$$

Considering now a slow modulation $\psi\left(\xi_{n}, \tau_{n}\right)$ in the variables (12), of the plane wave $A(n, t)$, we represent this modulation by the function $\psi(m, \tau)$ of the above defined discrete variable $m$ and of $\tau=\tau_{n}$. Then it is demonstrated in 20] that the first centered difference of $u_{n}(t)=A(n, t) \psi(m, \tau)$ reads

$$
\begin{aligned}
u_{n+1} & -u_{n-1}=\left[A_{n+1}-A_{n-1}\right] \psi_{m} \\
& +\left(\epsilon / v_{g}\right)\left[A_{n+1}+A_{n-1}\right] \partial_{\tau} \psi_{m} \\
& +\epsilon^{2} / 2\left[A_{n+1}+A_{n-1}\right]\left[\psi_{m+1}-\psi_{m-1}\right] \\
& +\left(\epsilon / v_{g}\right)^{2} \frac{1}{2}\left[A_{n+1}-A_{n-1}\right] \partial_{\tau}^{2} \psi_{m}+\mathcal{O}\left(\epsilon^{3}\right),
\end{aligned}
$$

while the second discrete derivative is given by

$$
\begin{aligned}
u_{n+1} & -2 u_{n}+u_{n-1}=\left[A_{n+1}-2 A_{n}+A_{n-1}\right] \psi_{m} \\
& +\left(\epsilon / v_{g}\right)\left[A_{n+1}-A_{n-1}\right] \partial_{\tau} \psi_{m} \\
& +\left(\epsilon / v_{g}\right)^{2} \frac{1}{2}\left[A_{n+1}+A_{n-1}\right] \partial_{\tau}^{2} \psi_{m} \\
& +\epsilon^{2} / 2\left[A_{n+1}-A_{n-1}\right]\left[\psi_{m+1}-\psi_{m-1}\right]+\mathcal{O}\left(\epsilon^{3}\right) .
\end{aligned}
$$

The discrete electrical lattice. We consider now the problem of the onset of modulational instability in the nonlinear electrical lattice experimentaly and theoreticaly studied in $[18$. The model reads

$$
\begin{array}{r}
\left(A+V_{n}\right) \partial_{t}^{2} V_{n}-\left(\partial_{t} V_{n}\right)^{2}= \\
\frac{u_{0}^{2}}{A}\left(A+V_{n}\right)^{2}\left[V_{n+1}+V_{n-1}-\left(2+\frac{\omega_{0}^{2}}{u_{0}^{2}}\right) V_{n}\right],
\end{array}
$$

where $A$ is a constant and $V_{n}(t)$ is the voltage of the $n$-th cell. The linearized version of this equation does possess the dispersion relation (2). The experiments consist in sending at one end of the electrical lattice a voltage $V_{0}(t)$ as a slightly modulated plane wave for various values of the carrier frequency and amplitude, and then measuring the output voltage at some cells along the lattice.

To proceed with discrete multiscaling, it is more convenient to work with the equivalent system 


$$
\begin{gathered}
\partial_{t} V_{n}=u_{0}\left[1+\frac{1}{A} V_{n}\right] B_{n} \\
\partial_{t} B_{n}=u_{0}\left[V_{n+1}+V_{n-1}-\left(2+\frac{\omega_{0}^{2}}{u_{0}^{2}}\right) V_{n}\right] .
\end{gathered}
$$

A perturbation expansion is now performed with the following series

$$
\begin{aligned}
& B_{n}=\sum_{p=1}^{\infty} \epsilon^{p} \sum_{\ell=-p}^{p} A^{\ell}(n, t) \psi_{p}^{(\ell)}(m, \tau), \\
& V_{n}=\sum_{p=1}^{\infty} \epsilon^{p} \sum_{\ell=-p}^{p} A^{\ell}(n, t) \phi_{p}^{(\ell)}(m, \tau),
\end{aligned}
$$

where of course $A^{\ell}(n, t)=\exp [i \ell(K n-\Omega t)]$, and where the reality condition results in $\psi_{p}^{(-\ell)}=\bar{\psi}_{p}^{(\ell)}$ and $\phi_{p}^{(-\ell)}=$ $\bar{\phi}_{p}^{(\ell)}$.

Inserting the above expressions in (18) and using the formula (16), we arrive at

$$
\begin{aligned}
& \phi_{1}^{(0)}=\psi_{1}^{(0)}=0, \quad \phi_{1}^{(1)}=\eta, \\
& \psi_{1}^{(1)}=-i \frac{\Omega}{u_{0}} \eta, \quad \phi_{2}^{(0)}=\psi_{2}^{(0)}=0, \\
& \phi_{2}^{(1)}=-\frac{i}{\Omega}\left[\partial_{\tau} \eta-u_{0} \delta\right], \quad \psi_{2}^{(1)}=\delta, \\
& \phi_{2}^{(2)}=-2 \frac{\Omega^{2}}{A a} \eta^{2}, \quad \psi_{2}^{(2)}=i \frac{\Omega}{u_{0}}\left[\frac{4 \Omega^{2}}{A a}+\frac{1}{A}\right] \eta^{2}, \\
& \phi_{3}^{(0)}=0, \quad \psi_{3}^{(0)}=-\frac{1}{A u_{0}} \partial_{\tau}|\eta|^{2},
\end{aligned}
$$

where $\eta=\eta_{m}(\tau)$ and $\delta=\delta_{m}(\tau)$ are the new unknowns and where we have defined

$$
a=-3 \omega_{0}^{2}-4 u_{0}^{2}(1-\cos K)^{2} .
$$

Solving then the system for $\phi_{3}^{(1)}$ and $\psi_{3}^{(1)}$, the unknown function $\delta_{m}(\tau)$ cancels out and we are left with the equation for $\eta_{m}(\tau)$ which eventually writes

$$
\frac{i}{2}\left[\eta_{m+1}-\eta_{m-1}\right]+Q \frac{\partial^{2} \eta_{m}}{\partial \tau^{2}}-\gamma\left|\eta_{m}\right|^{2} \eta_{m}=0,
$$

with the following $K$-dependent coefficients

$$
\begin{aligned}
& Q=\frac{1}{2} \frac{\partial^{2} K}{\partial \Omega^{2}}=\frac{u_{0}^{2}(1-\cos K)^{2}-\omega_{0}^{2} \cos K}{2 u_{0}^{4} \sin ^{3} K}, \\
& \gamma=\frac{1}{2 A^{2}} \frac{\Omega^{2}\left[\omega_{0}^{2}+4 u_{0}^{2} \sin ^{2} K\right]}{u_{0}^{2} \sin K\left[3 \omega_{0}^{2}+4 u_{0}^{2}(1-\cos K)^{2}\right]} .
\end{aligned}
$$

From (19) and (20) we have then

$$
V_{n}(t)=\epsilon \eta_{m}(\tau) e^{i(K n-\Omega t)}+\mathcal{O}\left(\epsilon^{2}\right)+c . c .
$$

Note that higher order corrections to $V(n, t)$ are either explicitely expressed in terms of $\eta$ through (20) or by linear inhomogeneous differential equations. That means that the theory is self-consistent and in particular that the overtones are by no means neglected.
Modulational instability. The continuous version of the equation (21) is a well known model for boundary value problems in optical fibers 21]. It has been studied also in the case of initial value problems in unstable media [7] to describe for instance Rayleigh-Taylor instability.

The plane wave solution to (21), say

$$
\eta_{m}(\tau)=B \exp [i(\lambda m-\nu \tau)],
$$

obeys the dispersion law

$$
\nu^{2}=-\frac{1}{Q}\left[\sin \lambda+\gamma|B|^{2}\right] .
$$

As we are considering a boundary value problem, $\nu$ and $B$ are the given frequency and amplitude of the modulation. Then, as $\sin (\lambda)$ is bounded, and this is precisely a discretreness effect (see conclusion), there is no real solution $\lambda$ if

$$
Q>0, \quad|B|^{2}>\frac{1}{\gamma}=B_{c r}^{2},
$$

leading to unstable plane waves.

Reporting to the definition (22) we first remark that $Q$ vanishes at $K=K_{c r}$ which is also the zero of the group velocity dispersion. Second, it is easy to see that

$$
K<K_{c r} \Rightarrow Q<0, \quad K>K_{c r} \Rightarrow Q>0 .
$$

Then the waves are unstable for $K>K_{c r}$ and $|B|>$ $\left|B_{c r}\right|$.

Application. The parameters of the experiments of [18] are

$$
\omega_{0}=2.13 \mathrm{MHz}, \quad u_{0}=1.77 \mathrm{MHz}, \quad A=3.9 \mathrm{~V} \text {, }
$$

for which the zero $K_{c r}$ of $\alpha(K)$ results to be

$$
K_{c r}=1.25 \text { rad.cell }^{-1} \text {, }
$$

in agreement with the experimental observation of [18].

Concerning the threshold amplitude $B_{c r}$ defined in (26), its actual value in the physical units is found by going from $\eta_{m}(\tau)$ to the value of $V_{n}(t)$ through (23), namely the critical value of the amplitude of $V_{n}(t)$ is simply $\epsilon B_{c r}$. Hence we must evaluate $\epsilon$ by using its very definition, the requirement of slow modulation (13). In one of the the experiments of 18] (see their fig. 6), an input plane wave of frequency $f=600 \mathrm{Khz}$ (wave number $K_{e}=2.15 \mathrm{rad} . c e l l^{-1}$ ) is modulated at $F=32 \mathrm{Khz}$ and sent in the lattice. Remembering the change of variables (12), in particular $\tau=\epsilon t$, such a modulation implies $\epsilon=f / F \sim 0.053$.

For the parameters (28), the critical amplitude $B_{c r}$, at wave number $K_{e}$ is easily evaluated and hence value of the instability threshold results to be

$$
\epsilon B_{c r}\left(K_{e}\right)=0.23 \mathrm{~V} \text {. }
$$

This value is significantly smaller than those currently used in the experiments of [18] where damping prevents 
the use of smaller voltage input. However, the actual dependence of $B_{c r}$ on the relative frequencies of the carrier and modulation waves (through the parameter $\epsilon$ ) allows one to vary the value of threshold $B_{c r}$. This property could be used to check the threshold amplitude on new experiments. As shown on the figure, we note finally that the function $B_{c r}(K)$ is bounded (maximum of $4.68 \mathrm{~V}$ at $K=2.59$ ), hence this threshold of instability exists at any carrier wave number in $] K_{c r}, \pi[$.

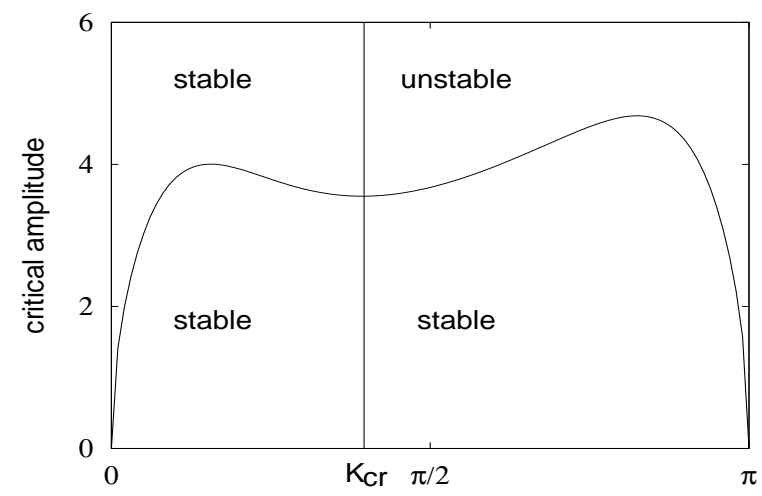

Fig.1: Critical amplitude $B_{c r}(K)$ in units of $\epsilon$

Predictions. The theory can also be applied to the sine-Gordon chain

$$
\ddot{u}_{n}-u_{0}^{2}\left(u_{n+1}-2 u_{n}+u_{n-1}\right)+\omega_{0}^{2} \sin u_{n}=0,
$$

which has the linear dispersion relation (2) and for which the limit model is 21) with the coefficient

$$
\gamma=\frac{\omega_{0}^{2}}{4 u_{0}^{2} \sin K}
$$

and of course the same value for $Q$. Performing the analysis shows an instability for wave numbers greater than the zero $K_{c r}$ of $Q(K)$, which develops for modulation amplitude obeying (in units of $\epsilon$ and in the Brillouin zone $K \in] 0, \pi[)$

$$
B>B_{c r}=\frac{\omega_{0}}{2 u_{0} \sqrt{\sin K}}
$$

For the integrable Toda lattice whose dispersion relation is $\Omega=2 \sin (K / 2)$, leading to the limit model (21) with 20

$$
Q=-\frac{1}{4} \frac{\sin (K / 2)}{\cos ^{3}(K / 2)}, \quad \gamma=-\frac{1}{\sin K},
$$

the theory implies that the waves are unstable for all $K$ and $|B|^{2}>|\sin K|$.

Conclusion. The instability described above results from wave scattering in a nonlinear medium (boundary value problem) which requires to use a change of variables like (12) which in turn leads to the unstable wave equation (21) for the envelope. The resulting instability is then of first order (a second perturbation analysis of the equation for the envelope is not required), and has for boundary value problems the same universal character as the Benjamin-Feir instability has for initial value problems.

Moreover this instability is a purely discrete phenomenon as indeed, performing a semi-discrete (discrete carrier and continuous envelope) analysis with the new variables (12) leads to the continous version of (21) and hence to the dispersion law

$$
\nu^{2}=-\frac{1}{Q}\left[\lambda+\gamma|B|^{2}\right]
$$

The point is that, even for positive values of $Q$, there always exists a real solution $\lambda$ of the above dispersion relation for any given modulation frequency $\nu$.

[1] T.B. Benjamin, J.E. Feir, J. Fluid Mech. 27, 417 (1967).

[2] V. I. Bespalov, V.I.Talanov, Sov. Phys. JETP Lett., 3, 307 (1966), [Pis'ma Zh. Exp. Teor. Fiz., 3, 471 (1966)].

[3] M. Remoissenet, Waves Called Solitons, Springer (Berlin 1996).

[4] T.B. Benjamin, Proc. Roy. Soc. London, A 328, 153 (1972).

[5] J.T. Stuart, R.C. Di Prima, Proc. Roy. Soc. Lond. A, 362, 27 (1978).

[6] N.N. Akhmediev, V.I. Korneev, Theor. Math. Phys., 69, 1089 (1986) [Teor. Mat. Fiz. (USSR), 69, 189 (1986)].

[7] M. Wadati, T. Iizuka, T. Yajima, Physica D51, 388 (1991)

[8] T. Taniuti, Prog. Theor. Phys. Suppl. 55, 1 (1974).

[9] R.S. MacKay, S. Aubry, Nonlinearity 7, 1623 (1994).

[10] S. Aubry, Physica D 103, 201 (1996).

[11] S. Flach, C.R. Willis, Phys. Rep. 295182 (1998).

[12] D. Hennig, G.P. Tsironis, Phys. Rep. 307, 333 (1999).

[13] Nonlinear Coherent Structures, M. Barthès and J. Leon (Eds.), Springer (Berlin 1990).

[14] Nonlinear Coherent Structures in Physics and Biology, K.H. Spatshek and F.G. Mertens (Eds.), Plenum (NewYork 1994).

[15] A. Tsurui, J. Phys. Soc. Japan, 34, 1462 (1973).

[16] S. Pnevmaticos, M. Remoissenet, N. Flytzanis, J. Phys. C, 16, L305 (1983); 18, 4603 (1985).

[17] P. Marquié, J.M. Bilbault, M. Remoissenet, Phys. Rev. E, 49, 828 (1994).

[18] P. Marquié, J.M. Bilbault, M. Remoissenet, Phys. Rev. E, 51, 6127 (1995).

[19] Yu.S. Kivshar, M. Peyrard, Phys. Rev. A 46, 3198 (1992).

[20] J. Leon, M. Manna, J. Phys. A: Math. Gen., 32, 2845 (1999).

[21] A. Hasegawa, F. Tappert, Appl. Phys. Lett. 23, 284 (1974). 